\title{
Pair density functional theory utilizing the noninteracting reference system: An effective initial theory
}

\author{
Masahiko Higuchi \\ Department of Physics, Faculty of Science, Shinshu University, Matsumoto 390-8621, Japan
}

Katsuhiko Higuchi

Graduate School of Advanced Sciences of Matter, Hiroshima University, Higashi-Hiroshima 739-8527, Japan

(Received 8 May 2008; revised manuscript received 4 August 2008; published 4 September 2008)

\begin{abstract}
We present a pair density (PD) functional scheme utilizing the noninteracting reference system. In order to check to what extent this scheme can express the correlation effects, actual calculations are performed for the neutral neon atom. It is shown that this scheme reproduces about $20 \%$ of the correlation energy through use of noninteracting single determinants with an approximate correlating kinetic energy functional. Thus, since it obviously provides the $N$-representable and correlated PD, this scheme can be positioned as an effective initial theory in the field of the PD functional theory, just like the position of the Hartree-Fock approximation in the field of the wave function theory.
\end{abstract}

DOI: 10.1103/PhysRevB.78.125101

PACS number(s): 71.15.Mb

\section{INTRODUCTION}

In recent years, we have proposed the extended constrained-search (ECS) theory in which arbitrary physical quantities can be chosen as basic variables. ${ }^{1-5}$ The ECS theory has a strong merit to reproduce, in principle rigorously, the physical quantities that characterize the groundstate properties of the intended system. The validity of the ECS theory has been confirmed by revisiting the previous theories such as the spin density-functional theory, current density-functional theory, local density approximation plus Hubbard $U(\mathrm{LDA}+U)$ method, etc. ${ }^{1-5}$

As the next step toward developing the effective theory, the choice of basic variables of the ECS theory is our matter of interest. As a candidate, we can immediately hit on the pair density (PD) that corresponds to the diagonal element of the spinless second-order reduced density matrix. The PD includes more information than the electron density, or to be more precise, the expectation value of the arbitrary twoparticle operator can be expressed rigorously by using the PD. ${ }^{6,7}$ Therefore, this type of the ECS theory is expected to provide a more detailed description for the electron correlation. Along this line, we have recently proposed a PD functional theory utilizing the noninteracting reference system. ${ }^{8}$ Although it is a preliminary work, we have confirmed that the resultant single-particle equation is analogous to the Hartree-Fock (HF) equation but contains the correlation terms definitely. ${ }^{8}$

Of course, due to the above-mentioned merit of the PD, a lot of works concerning the PD functional theory have been done. ${ }^{8-24}$ They are expected to provide a possible way to go beyond the conventional density-functional theory, ${ }^{25,26}$ and have gotten a lot of attention recently. The PD functional theory is yet a developing field. We can say that our scheme ${ }^{8}$ is one of these developing PD functional theories.

In this paper, we shall evaluate the validity of this scheme from the quantitative point of view by performing actual calculations for the neutral neon atom. The results show that this scheme can be positioned as an effective initial theory in the field of the PD functional theory because it obviously provides the $N$-representable and correlated PD, and because there hardly exists the computational PD functional scheme so far. This position is just analogous to that of the HF approximation among many kinds of wave function theories. ${ }^{27,28}$ The details will be discussed in the following sections.

Organization of this paper is as follows. In Sec. II, we present the outline of the PD functional theory utilizing the noninteracting reference system. In Sec. III, features of this scheme are discussed from the formal viewpoint. Several formal merits are listed. In order to give a direct proof of the validity of the present scheme, we perform the test calculations for the neutral neon atom in Sec. IV. The results are quite interesting and show that the present scheme is potentially an effective starting point of the PD functional theory. This positioning will be discussed in detail in Sec. V. Finally, some concluding remarks are given in Sec. VI.

\section{PD FUNCTIONAL THEORY USING THE NONINTERACTING REFERENCE SYSTEM}

First of all, let us review our PD functional theory ${ }^{8}$ for later convenience. Our scheme is based on the ECS theory. ${ }^{1-5}$ We shall consider a system, the Hamiltonian of which is given by

$$
\hat{H}=\hat{T}+\hat{W}+\int \hat{\rho}(\mathbf{r}) v_{\text {ext }}(\mathbf{r}) \mathrm{d} \mathbf{r},
$$

where $\hat{T}, \hat{W}$, and $\hat{\rho}(\mathbf{r})$ are operators of the kinetic energy, electron-electron interaction, and electron density, respectively, and where $v_{\text {ext }}(\mathbf{r})$ stands for the external potential. The $\mathrm{PD}$ is defined as

$$
\begin{aligned}
\gamma^{(2)}\left(\mathbf{r r}^{\prime} ; \mathbf{r} \mathbf{r}^{\prime}\right)= & \frac{1}{2}\langle\Psi| \iint \hat{\psi}^{\dagger}(\mathbf{r}, \eta) \hat{\psi}^{\dagger}\left(\mathbf{r}^{\prime}, \eta^{\prime}\right) \hat{\psi}\left(\mathbf{r}^{\prime}, \eta^{\prime}\right) \hat{\psi}(\mathbf{r}, \eta) \\
& \times \mathrm{d} \eta \mathrm{d} \eta^{\prime}|\Psi\rangle,
\end{aligned}
$$

where $\hat{\psi}(\mathbf{r}, \eta)$ and $\hat{\psi}^{\dagger}(\mathbf{r}, \eta)$ are field operators of electrons, 
and $\mathbf{r}$ and $\eta$ are spatial and spin coordinates, respectively, and $\Psi$ is the antisymmetric wave function. The universal functional that is independent of the external potential is given by

$$
\begin{aligned}
F\left[\gamma^{(2)}\right] & =\underset{\Psi \rightarrow \gamma^{(2)}}{\operatorname{Min}}\langle\Psi|\hat{T}+\hat{W}| \Psi\rangle \\
& =T\left[\gamma^{(2)}\right]+e^{2} \iint \frac{\gamma^{(2)}\left(\mathbf{r r}^{\prime} ; \mathbf{r r}^{\prime}\right)}{\left|\mathbf{r}-\mathbf{r}^{\prime}\right|} \mathrm{d} \mathbf{r} \mathrm{d} \mathbf{r}^{\prime}
\end{aligned}
$$

with

$$
T\left[\gamma^{(2)}\right]=\underset{\Psi \rightarrow \gamma^{(2)}}{\operatorname{Min}}\langle\Psi|\hat{T}| \Psi\rangle,
$$

where $\Psi \rightarrow \gamma^{(2)}$ denotes the searching over all antisymmetric wave functions that yield a prescribed $\gamma^{(2)}\left(\mathbf{r} \mathbf{r}^{\prime} ; \mathbf{r r}^{\prime}\right)$, and $T\left[\gamma^{(2)}\right]$ is the kinetic energy functional. Using this universal functional, the Hohenberg-Kohn theorem can be proven in the extended form. ${ }^{1,8,9,15}$ The variational principle of this theorem is given by

$$
E_{0}=\underset{\gamma^{(2)}}{\operatorname{Min}} E_{v_{\mathrm{ext}}}\left[\gamma^{(2)}\right]
$$

with

$$
E_{v_{\mathrm{ext}}}\left[\gamma^{(2)}\right]=F\left[\gamma^{(2)}\right]+\frac{2}{N-1} \iint \gamma^{(2)}\left(\mathbf{r} \mathbf{r}^{\prime} ; \mathbf{r r}^{\prime}\right) v_{\mathrm{ext}}(\mathbf{r}) \mathrm{d} \mathbf{r} \mathrm{d} \mathbf{r}^{\prime}
$$

where $E_{0}$ denotes the ground-state energy. In order to perform this variational principle, we need to define the search region within the $N$-representable PDs. However, the necessary and sufficient conditions for the $N$-representable PD have not yet been given in a practical form. ${ }^{6,7,29-45}$ Our strategy is to restrict the search region within the set of PDs that are constructed from the single Slater determinants (SSDs). It prevents the solution from being unphysical, in other words, it guarantees the solution to be necessarily $N$-representable. This is one of the strong merits of the present scheme. But in the meanwhile, there exists a problem such that the solution may be far from the correct groundstate PD. This is because the search region of the present scheme is smaller than the set of whole $N$-representable PDs. This problem is, of course, related to the necessary and sufficient conditions for the $N$-representable PD, which will be discussed in Sec. $\mathrm{V}$ in relation to the positioning of the present scheme.

According to the strategy mentioned above, we introduce the noninteracting reference system so as to reproduce the PD. The kinetic energy functional of the reference system is defined as

$$
T_{s}\left[\gamma^{(2)}\right]=\underset{\Phi \rightarrow \gamma^{(2)}}{\operatorname{Min}}\langle\Phi|\hat{T}| \Phi\rangle,
$$

where $\Phi$ is the SSD. Using $T_{s}\left[\gamma^{(2)}\right]$, we shall give $F\left[\gamma^{(2)}\right]$ as the following form:

$$
F\left[\gamma^{(2)}\right]=T_{s}\left[\gamma^{(2)}\right]+\Delta T_{c}\left[\gamma^{(2)}\right]+e^{2} \iint \frac{\gamma^{(2)}\left(\mathbf{r} \mathbf{r}^{\prime} ; \mathbf{r r}^{\prime}\right)}{\left|\mathbf{r}-\mathbf{r}^{\prime}\right|} \mathrm{d} \mathbf{r} \mathrm{d} \mathbf{r}^{\prime},
$$

where $\Delta T_{c}\left[\gamma^{(2)}\right]$ is the correlating kinetic energy that is defined by

$$
\Delta T_{c}\left[\gamma^{(2)}\right]=T\left[\gamma^{(2)}\right]-T_{s}\left[\gamma^{(2)}\right] .
$$

Substituting Eq. (8) into Eq. (6), we get

$$
\begin{aligned}
E_{v_{\mathrm{ext}}}\left[\gamma^{(2)}\right]= & T_{s}\left[\gamma^{(2)}\right]+\Delta T_{c}\left[\gamma^{(2)}\right]+e^{2} \iint \frac{\gamma^{(2)}\left(\mathbf{r} \mathbf{r}^{\prime} ; \mathbf{r r}^{\prime}\right)}{\left|\mathbf{r}-\mathbf{r}^{\prime}\right|} \mathrm{d} \mathbf{r} \mathrm{d} \mathbf{r}^{\prime} \\
& +\frac{2}{N-1} \iint \gamma^{(2)}\left(\mathbf{r} \mathbf{r}^{\prime} ; \mathbf{r r}^{\prime}\right) v_{\mathrm{ext}}(\mathbf{r}) \mathrm{d} \mathbf{r} \mathrm{d} \mathbf{r}^{\prime} .
\end{aligned}
$$

The variational principle yields the following single-particle equation:

$$
\begin{aligned}
& \left\{-\frac{\hbar^{2} \nabla^{2}}{2 m}+v_{\mathrm{ext}}(\mathbf{r})\right\} \phi_{\xi}(\mathbf{r}, \eta) \\
& \quad+\iint_{\mathrm{d}} \mathrm{d} \mathbf{r}^{\prime} \mathrm{d} \eta^{\prime}\left\{\frac{e^{2}}{\left|\mathbf{r}-\mathbf{r}^{\prime}\right|}+\frac{1}{2} \frac{\delta \Delta T_{c}\left[\gamma^{(2)}\right]}{\delta \gamma^{(2)}\left(\mathbf{r r}^{\prime} ; \mathbf{r} \mathbf{r}^{\prime}\right)}+\frac{1}{2} \frac{\delta \Delta T_{c}\left[\gamma^{(2)}\right]}{\delta \gamma^{(2)}\left(\mathbf{r}^{\prime} \mathbf{r} ; \mathbf{r}^{\prime} \mathbf{r}\right)}\right\} \\
& \quad \times\left\{\sum_{\nu} \phi_{\nu}^{*}\left(\mathbf{r}^{\prime}, \eta^{\prime}\right) \phi_{\nu}\left(\mathbf{r}^{\prime}, \eta^{\prime}\right) \phi_{\xi}(\mathbf{r}, \eta)-\sum_{\nu} \phi_{\nu}^{*}\left(\mathbf{r}^{\prime}, \eta^{\prime}\right) \phi_{\xi}\left(\mathbf{r}^{\prime}, \eta^{\prime}\right) \phi_{\nu}(\mathbf{r}, \eta)\right\}=\varepsilon_{\xi} \phi_{\xi}(\mathbf{r}, \eta),
\end{aligned}
$$

where $\phi_{\zeta}(\mathbf{r}, \eta)$ are the constituent orbitals of the SSD. The explicit form of the PD reproduced in the reference system is given by

$$
\gamma^{(2)}\left(\mathbf{r r}^{\prime} ; \mathbf{r r}^{\prime}\right)=\frac{1}{2} \sum_{\mu, \nu} \iint\left\{\phi_{\mu}^{*}(\mathbf{r}, \eta) \phi_{\nu}^{*}\left(\mathbf{r}^{\prime}, \eta^{\prime}\right) \phi_{\mu}(\mathbf{r}, \eta) \phi_{\nu}\left(\mathbf{r}^{\prime}, \eta^{\prime}\right)-\phi_{\mu}^{*}(\mathbf{r}, \eta) \phi_{\nu}^{*}\left(\mathbf{r}^{\prime}, \eta^{\prime}\right) \phi_{\nu}(\mathbf{r}, \eta) \phi_{\mu}\left(\mathbf{r}^{\prime}, \eta^{\prime}\right)\right\} \mathrm{d} \eta \mathrm{d} \eta^{\prime}
$$


In order to solve the single-particle equation [Eq. (11)], the approximate form of $\Delta T_{c}\left[\gamma^{(2)}\right]$ is absolutely necessary. Utilizing the scaling properties of the kinetic energy functionals, ${ }^{8,13}$ we develop an approximate form

$$
\Delta T_{c}\left[\gamma^{(2)}\right]=K \iint \mathrm{d} \mathbf{r} \mathrm{d} \mathbf{r}^{\prime} \frac{\gamma^{(2)}\left(\mathbf{r} \mathbf{r}^{\prime} ; \mathbf{r r}^{\prime}\right)^{7 / 6}}{\left|\mathbf{r}-\mathbf{r}^{\prime}\right|},
$$

where $K$ is the arbitrary constant. Note that an approximate form for $T\left[\gamma^{(2)}\right]$ that is analogous to Eq. (13) appears in Ref. 13. The derivation of Eq. (13) is shown in Appendix A. The PD can be obtained by solving Eqs. (11)-(13) in a selfconsistent way.

\section{FORMAL FEATURES OF THE PRESENT SCHEME}

We can confirm the merits of the present scheme even within a formal discussion. Before quantitative evaluation of the present scheme, let us list the merits of the present scheme from a formal viewpoint.

In the PD functional theory, there exist two well-known problems ${ }^{8}$ i.e., (i) $N$-representability of the PD, and (ii) approximate form of the kinetic energy functional. The first one is due to the fact that the necessary and sufficient conditions for the $N$-representable PD are not yet known in a practical form, and the second one is that the kinetic energy cannot exactly be written by using the PD alone. As shown above, the present scheme is obviously free from the $N$-representability problem and makes significant progress concerning the second problem. That is to say,

(a) The PD is guaranteed to be necessarily $N$-representable by constructing the PD from the SSD.

(b) An approximate form of the kinetic energy functional can be easily derived by utilizing the scaling properties of the kinetic energy functionals. This is also due to the introduction of the noninteracting reference system.

In addition to these merits, we can point out the other features as follows:

(c) As can be seen in Eq. (11), the single-particle equation is similar to the HF equation but definitely contains the correlation terms. Namely, the fourth and fifth terms of the lefthand side are the additional terms to the HF equation.

(d) The total energy of this scheme is shown to be lower than that of the HF equation. This means that the present scheme includes the correlation effects definitely. The proof is given in Appendix B.

(e) The present scheme is consistent with the fact that correlation effects have a tendency to raise the kinetic energy as compared to the HF kinetic energy. ${ }^{46}$ This can be understood as follows: According to the virial theorem, correlation effects raise the kinetic energy, which means that $T\left[\gamma^{(2)}\right]$ $\geq T_{\mathrm{HF}}$, where $T_{\mathrm{HF}}$ is the HF kinetic energy. On the other hand, the inequality $\Delta T_{c}\left[\gamma^{(2)}\right]\left(=T\left[\gamma^{(2)}\right]-T_{s}\left[\gamma^{(2)}\right]\right) \leq 0$ exactly holds. ${ }^{8}$ Therefore, we can conclude $T_{s}\left[\gamma^{(2)}\right] \geq T\left[\gamma^{(2)}\right] \geq T_{\mathrm{HF}}$. The resultant inequality $T_{s}\left[\gamma^{(2)}\right] \geq T_{\mathrm{HF}}$ is consistent with the fact that the kinetic energy increases beyond the HF kinetic energy due to correlation effects.

Thus, it is formally shown that the present scheme provides the $N$-representable and correlated PD. However, as already mentioned, there exists a remaining problem that the search region is smaller than the set of all of $N$-representable PDs. Although the correlation terms are included in the single-particle equation formally, it is difficult to judge to what extent the correlation effects can be covered by the present scheme. The direct and best way to answer this question is to perform actual calculations. The formal merits mentioned above become substantial only after checking the validity of the present scheme quantitatively.

\section{TEST CALCULATIONS OF THE PRESENT SCHEME}

We shall perform the actual calculations for neutral neon atom so as to check the performance of the present scheme quantitatively. In actual calculations, how to determine the value of $K$ in Eq. (13) is an important issue because the magnitude of correlation effects directly depends on it. In the present calculations, it is determined by requiring the electron density to fit the result of the configuration interaction (CI) calculation. This determination method is reasonable because the search region of the present scheme necessarily includes the set of PDs that yield the correct electron density. This can be understood if we note that all of $N$-representable electron densities, the set of which is identical with that of SSD-representable electron densities, ${ }^{47}$ can be obtained by integrating SSD-representable PDs. The value of $K$ used here will be shown later, together with calculation results.

In the present calculations, the spherical (central-field) approximation is adopted to solve the single-particle equation [Eq. (11)]. This approximation is conventionally applied to atomic structure calculations using the HF equation or KohnSham equation, or other single-particle equation. ${ }^{48}$ In what follows, we will rewrite Eq. (11) on the basis of the spherical approximation. ${ }^{49-54}$

A central-field wave function is of the form

$$
\phi_{\xi}(x)=\frac{P_{n l}(r)}{r} Y_{l m}(\hat{r}) \chi_{\sigma}(\eta),
$$

where $P_{n l}(r), Y_{l m}(\hat{r})$, and $\chi_{\sigma}(\eta)$ denote the radial wave function multiplied by $r$, spherical harmonics and spin function, respectively. Substituting Eqs. (13) and (14) into Eq. (11), and acting with the operator $\sum_{m=-l}^{l} \sum_{\sigma} \int \mathrm{d} \hat{r} \int \mathrm{d} \eta Y_{l m}^{*}(\hat{r}) \chi_{\sigma}^{*}(\eta)$ on both sides of Eq. (11), we have

$$
\left\{-\frac{\hbar^{2}}{2 m} \frac{\mathrm{d}^{2}}{\mathrm{~d} r^{2}}+\frac{\hbar^{2} l(l+1)}{2 m r^{2}}+\frac{Z e^{2}}{r}+e^{2} \sum_{n^{\prime} l^{\prime}}^{\text {occ. }} N_{n^{\prime} l^{\prime}} \int \frac{1}{r_{>}}\left|P_{n^{\prime} l^{\prime}}\left(r^{\prime}\right)\right|^{2} \mathrm{~d} r^{\prime}-\varepsilon_{n l}\right\} P_{n l}(r)=-F_{n l}(r)
$$

with 


$$
\begin{aligned}
F_{n l}(r)= & -\frac{e^{2}}{2} \sum_{n^{\prime} l^{\prime}}^{\text {occ. }} \sum_{\lambda=\left|l-l^{\prime}\right|}^{l+l^{\prime}} N_{n^{\prime} l^{\prime}}\left(\begin{array}{lll}
l & l^{\prime} & \lambda \\
0 & 0 & 0
\end{array}\right)^{2} P_{n^{\prime} l^{\prime}}(r) \int_{0}^{\infty} \frac{r_{<}^{\lambda}}{r_{>}^{\lambda+1}} P_{n^{\prime} l^{\prime}}^{*}\left(r^{\prime}\right) P_{n l}\left(r^{\prime}\right) \mathrm{d} r^{\prime}+\frac{7 K}{2^{1 / 6} \cdot 6} \theta^{1}(r) \rho_{0}(r)^{1 / 6} P_{n l}(r) \\
& -\frac{7 K}{2^{1 / 6} \cdot 12} \sum_{n^{\prime} l^{\prime}} \sum_{\lambda=\left|l-l^{\prime}\right|}^{\text {occ. }} N_{n^{\prime} l^{\prime}}\left(\begin{array}{lll}
l & l^{\prime} & \lambda \\
0 & 0 & 0
\end{array}\right)^{2} \theta_{n l, n^{\prime} l^{\prime}, \lambda}^{2}(r) \rho_{0}(r)^{1 / 6} P_{n^{\prime} l^{\prime}}(r) \\
& -\frac{7 K}{2^{1 / 6} \cdot 72} \sum_{n_{1} l_{1}} \sum_{n_{2} l_{2}} \sum_{L=\left|l_{1}-l_{2}\right|}^{l_{1} \mid l_{2}} N_{n_{1} l_{1} l_{1}} N_{n_{2} l_{2}}\left(\begin{array}{lll}
l_{1} & l_{2} & L \\
0 & 0 & 0
\end{array}\right)^{2} \theta_{n_{1} l_{1}, n_{2} l_{2}, L}^{2}(r) \frac{\rho_{0}(r)^{-5 / 6}}{4 \pi r^{2}} P_{n_{1} l_{1}}^{*}(r) P_{n_{2} l_{2}}(r) P_{n l}(r) \\
& +\frac{7 K}{2^{1 / 6} \cdot 144} \sum_{n^{\prime} l^{\prime}} \sum_{n_{1} l_{1}} \sum_{n_{2} l_{2}} \sum_{L=\left|l_{1}-l_{2}\right|} \sum_{\lambda=\left|l-l^{\prime}\right|} \sum_{L^{\prime}=|L-\lambda|}^{L c c \mid} N_{n_{1} l_{1}} N_{n_{2} l_{2}} N_{n^{\prime} l^{\prime}}\left(\begin{array}{ccc}
l_{1} & l_{2} & L \\
0 & 0 & 0
\end{array}\right)^{2}\left(\begin{array}{lll}
l & l^{\prime} & \lambda \\
0 & 0 & 0
\end{array}\right)^{2}\left(\begin{array}{ccc}
L & \lambda & L^{\prime} \\
0 & 0 & 0
\end{array}\right)^{2} \\
& \times(2 L+1)(2 \lambda+1) \theta_{n l, n_{1} l_{1}, n_{2} l_{2}, n^{\prime} l^{\prime}, L^{\prime}}^{3}(r) \frac{\rho_{0}(r)^{-5 / 6}}{4 \pi r^{2}} P_{n_{1} l_{1}}^{*}(r) P_{n_{2} l_{2}}(r) P_{n^{\prime} l^{\prime}}(r),
\end{aligned}
$$

where

$$
\begin{gathered}
\theta^{1}(r)=\int_{0}^{\infty} \frac{4 \pi r^{\prime 2}}{r_{>}} \rho_{0}\left(r^{\prime}\right)^{7 / 6} \mathrm{~d} r^{\prime} \\
\theta_{n_{1} l_{1}, n_{2} l_{2}, L}^{2}(r)=\int_{0}^{\infty} \frac{r_{<}^{L}}{r_{>}^{L+1}} \rho_{0}\left(r^{\prime}\right)^{1 / 6} P_{n_{2} l_{2}}^{*}\left(r^{\prime}\right) P_{n_{1} l_{1}}\left(r^{\prime}\right) \mathrm{d} r^{\prime} \\
\theta_{n l, n_{1} l_{1}, n_{2} l_{2}, n^{\prime} l^{\prime}, L^{\prime}}^{3}(r)=\int_{0}^{\infty} \frac{r_{<}^{L^{\prime}}}{r_{>}^{L^{\prime}+1}} \frac{\rho_{0}\left(r^{\prime}\right)^{-5 / 6}}{4 \pi r^{\prime 2}} P_{n_{2} l_{2}}^{*}\left(r^{\prime}\right) P_{n^{\prime} l^{\prime}}^{*}\left(r^{\prime}\right) \\
\\
\times P_{n_{1} l_{1}}\left(r^{\prime}\right) P_{n l}\left(r^{\prime}\right) \mathrm{d} r^{\prime} .
\end{gathered}
$$

Here, $N_{n l}$ denotes the occupation number of the $n l$-shell, and takes on integer values between 0 and $2 l(l+1)$. The bracket

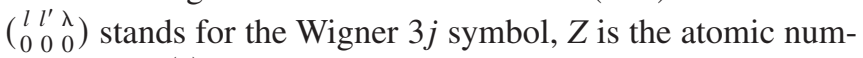
ber, and $\rho_{0}(r)$ denotes the ground-state electron density. In the above derivation, $\gamma^{(2)}\left(\mathbf{r} \mathbf{r}^{\prime} ; \mathbf{r r}^{\prime}\right)^{p}$ is approximately calculated by the following formula:

$$
\begin{aligned}
\gamma^{(2)}\left(\mathbf{r} \mathbf{r}^{\prime} ; \mathbf{r r}^{\prime}\right)^{p} & =\left\{a\left(\mathbf{r}, \mathbf{r}^{\prime}\right)+b\left(\mathbf{r}, \mathbf{r}^{\prime}\right)\right\}^{p} \\
& \simeq a\left(\mathbf{r}, \mathbf{r}^{\prime}\right)^{p}+p a\left(\mathbf{r}, \mathbf{r}^{\prime}\right)^{p-1} b\left(\mathbf{r}, \mathbf{r}^{\prime}\right),
\end{aligned}
$$

where $a\left(\mathbf{r}, \mathbf{r}^{\prime}\right)$ and $b\left(\mathbf{r}, \mathbf{r}^{\prime}\right)$ correspond to the first and second terms of Eq. (12), respectively, and $p$ is a positive number. This approximation is reasonable because the second term is expected to be smaller than the first one.

Equation (15) is the radial differential equation that should be solved in a self-consistent way. The fourth term in the left-hand side of Eq. (15) is the classical Coulomb potential, and the first term in the right-hand side of Eq. (16) is the Fock potential (exchange potential). Of course, these two terms have the same form as the corresponding potentials in the HF equation. ${ }^{48}$ The last four terms in right-hand side of Eq. (16) correspond to the correlation potentials that newly appear in the present scheme.

As for the total energy, applying the spherical approximation, we have

$$
\begin{aligned}
E_{\text {total }}= & -\sum_{n l}^{\text {occ. }} N_{n l} \int F_{n l}(r) P_{n l}^{*}(r) \mathrm{d} r-\frac{e^{2}}{2} \sum_{n^{\prime} l^{\prime}}^{\text {occ. }} N_{n^{\prime} l^{\prime}} \iint \frac{4 \pi r^{2}\left|P_{n^{\prime} l^{\prime}}\left(r^{\prime}\right)\right|^{2}}{r_{>}} \rho_{0}(r) \mathrm{d} r^{\prime} \mathrm{d} r+\sum_{n l} N_{n l^{\prime}} \varepsilon_{n l}-\frac{1}{4} \sum_{n l}^{\text {occ. occ. }} \sum_{n^{\prime} l^{\prime}} \sum_{\lambda=\left|l-l^{\prime}\right|}^{l+l^{\prime}} N_{n l^{\prime}} N_{n^{\prime} l^{\prime}} \\
& \times\left(\begin{array}{lll}
l & l^{\prime} & \lambda \\
0 & 0 & 0
\end{array}\right)^{2} I_{n l, n^{\prime} l^{\prime}}^{\lambda}+\frac{K}{2^{7 / 6}} \int 4 \pi r^{2} \rho_{0}(r)^{7 / 6} \theta^{1}(r) \mathrm{d} r-\frac{K}{2^{7 / 6} \cdot 12} \sum_{n l} \sum_{n^{\prime} l^{\prime}} \sum_{\lambda=\left|l-l^{\prime}\right|}^{\text {occ. occ. }} N_{n l^{\prime}+l^{\prime}} N_{n^{\prime} l^{\prime}}\left(\begin{array}{lll}
l & l^{\prime} & \lambda \\
0 & 0 & 0
\end{array}\right)^{2} \\
& \times \int \rho_{0}(r)^{1 / 6} P_{n l}^{*}(r) P_{n^{\prime} l^{\prime}}(r) \theta_{n l, n^{\prime} l^{\prime}, \lambda}^{2}(r) \mathrm{d} r
\end{aligned}
$$




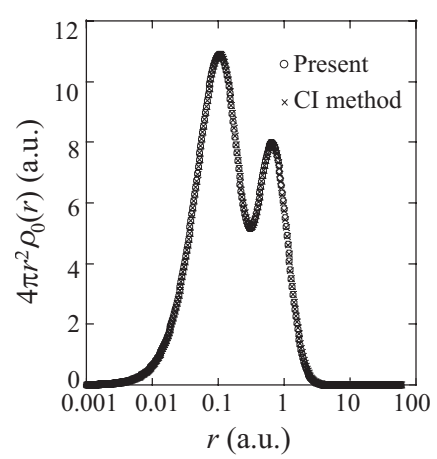

FIG. 1. Profiles of the electron density calculated by the present scheme (open circles) and the CI method (crosses) (Ref. 55) in atomic unit (a.u.). The value of $K$ is set to $-1.6 \times 10^{-3}$ in the present calculation.

with

$$
I_{n l, n^{\prime} l^{\prime}}^{\lambda}=\iint P_{n l}^{*}(r) P_{n^{\prime} l^{\prime}}^{*}\left(r^{\prime}\right) \frac{r_{<}^{\lambda}}{r_{>}^{\lambda+1}} P_{n^{\prime} l^{\prime}}(r) P_{n l}\left(r^{\prime}\right) \mathrm{d} r \mathrm{~d} r^{\prime} .
$$

Here we have used the approximate formula Eq. (20) in calculating $\Delta T_{c}\left[\gamma^{(2)}\right]$.

Equation (15) is solved numerically for neutral neon atom by using the standard Green's function technique. The convergence is checked by using both the electron density and the total energy [Eq. (21)]. Additional terms (i.e., correlation potentials) are not complicated, so that computation time is comparable to that of HF equation. This is also a strong merit of the present scheme.

We now turn to numerical results. First, the atomic structure calculations are performed with various values of $K$ so as to search the best one by means of the least square method. Figure 1 shows the profile of the electron density that is calculated with $K=-1.6 \times 10^{-3}$, together with the corresponding profile calculated by the CI method. ${ }^{55}$ The best agreement is achieved at $K=-1.6 \times 10^{-3}$ with the root-meansquare error of 0.04 . This negative value is consistent with the fact that $\Delta T_{c}\left[\gamma^{(2)}\right]$ should be negative [see the feature (e) in Sec. III]. The calculation results using $K=-1.6 \times 10^{-3}$ are summarized in Table I. The total energy of the present scheme is reasonably lower than that of the HF approximation (-257.094 Ry.) (Ref. 56) and higher than that of the CI method (-257.856 Ry.). ${ }^{57}$ Also, the present scheme can cover about $20 \%$ of the electron correlation from the viewpoint of the correlation energy. These are not negligibly small, and are the direct answers to the question: "To what extent can the correlation effects be covered by the present scheme?"

\section{POSITION OF THE PRESENT SCHEME}

On the basis of results and discussions thus shown, we shall clarify the position of the present scheme in the field of the PD functional theory. First, let us consider the position of the HF approximation among the wave function theories. As is well known, the HF approximation gives the best approximation of the ground state within the set of the SSDs. In other words, the best solution is searched within the restricted set of antisymmetric wave functions. Similarly to the HF approximation, the present scheme gives the best approximation of the ground-state PD within the restricted set of $N$-representable PDs that arise from single determinants. Thus, the position of the present scheme in the field of the PD functional theory just corresponds to that of the HF approximation in the field of the wave function theory.

There is no doubt that the HF approximation is an effective initial scheme in the field of the wave function theory. A variety of the wave function theories have been developed on the basis of the HF approximation. ${ }^{27,28}$ Likewise, it is expected that the present scheme would give an appropriate starting point of the PD functional theory. This expectation has, actually and definitely, been verified by the present test calculations. Namely, we have confirmed that the present scheme obviously provides the $N$-representable and correlated PD, and can cover the correlation effects by about $20 \%$ quantitatively. It can therefore be said that the present scheme is positioned as an effective initial one in the field of the PD functional theory.

\section{CONCLUDING REMARKS}

We present the PD functional theory utilizing the noninteracting reference system, and show by the numerical calculations that it obviously provides the $N$-representable and correlated PD. It is also shown that the position of the present scheme in the field of the PD functional theory just corresponds to that of the HF approximation in the field of the wave function theory. In other words, the present scheme can be positioned as an effective initial scheme in the field of the PD functional theory. This is quite important because the development of the PD functional theory utilizing this initial theory as a starting point will be anticipated, as the wave function theory has been developed on the basis of the HF approximation so far.

TABLE I. Atomic structure calculations for neutral neon atom.

\begin{tabular}{lcccc}
\hline \hline & $K$ & RMSE $^{\mathrm{a}}$ & Total energy (Ry) & Correlation energy (Ry) \\
\hline Present & $-1.6 \times 10^{-3}$ & 0.04 & -257.268 & -0.174 \\
HF & 0.0 & 0.24 & -257.094 & $-0.762^{\mathrm{b}}$ \\
CI method & & & $-257.856^{\mathrm{b}}$ & - \\
\hline \hline
\end{tabular}

Root-mean-square error for the electron density.

${ }^{\mathrm{b}}$ Reference 57 . 
Besides the above-mentioned developments based on the initial scheme, it would be also interesting to combine the present scheme with the recent works such as those in Refs. 39 and 45 . These works are related to the present scheme in a sense that they are based on the Kohn-Sham "orbital model" plus a PD correction. For example, one can try one of the Levy-Ayers functionals ${ }^{39}$ in conjunction with the present scheme.

Although the PD functional theory is a very hot area of the energy-band theory in recent years, there hardly exists the computational PD functional scheme so far. The present scheme would be the valid one in the sense that it is computational and contains the correlation effects definitely.

\section{ACKNOWLEDGMENTS}

This work was partially supported by Grant-in-Aid for Scientific Research (Grant No. 19540399) and for Scientific Research in Priority Areas (Grant No. 17064006) of The Ministry of Education, Culture, Sports, Science, and Technology, Japan.

\section{APPENDIX A: DERIVATION OF Eq. (13)}

In this appendix, the approximate form Eq. (13) is derived by using the scaling relation of $\Delta T_{c}\left[\gamma^{(2)}\right]$. The derivation procedure is similar to that of the alternative approximate form that has been presented in the previous paper. ${ }^{8}$ Let us start with the scaling relation of $\Delta T_{c}\left[\gamma^{(2)}\right]$. We have $\mathrm{e}^{8,13}$

$$
\Delta T_{c}\left[\gamma_{\zeta}^{(2)}\right]=\zeta^{2} \Delta T_{c}\left[\gamma^{(2)}\right]
$$

Here $\gamma_{\zeta}^{(2)}\left(\mathbf{r r}^{\prime} ; \mathbf{r r}^{\prime}\right)$ stands for the PD that is calculated by the scaled wave function, which is given by

$$
\gamma_{\zeta}^{(2)}\left(\mathbf{r} \mathbf{r}^{\prime} ; \mathbf{r} \mathbf{r}^{\prime}\right)=\zeta^{6} \gamma^{(2)}\left(\zeta \mathbf{r} \zeta \mathbf{r}^{\prime} ; \zeta \mathbf{r} \zeta \mathbf{r}^{\prime}\right) .
$$

Acting with $\lim _{\zeta \rightarrow 1} \frac{\partial}{\partial \zeta}$ on both sides of Eq. (A1), and utilizing the integration by parts, we obtain

$$
\begin{aligned}
2 \Delta T_{c}\left[\gamma^{(2)}\right]= & -\iint \gamma^{(2)}\left(\mathbf{r} \mathbf{r}^{\prime} ; \mathbf{r r}^{\prime}\right) \\
& \times \mathbf{r} \cdot \nabla\left\{\frac{\delta \Delta T_{c}\left[\gamma^{(2)}\right]}{\delta \gamma^{(2)}\left(\mathbf{r r}^{\prime} ; \mathbf{r r}^{\prime}\right)}\right\} \mathrm{d} \mathbf{r} \mathrm{d} \mathbf{r}^{\prime} \\
& -\iint \gamma^{(2)}\left(\mathbf{r} \mathbf{r}^{\prime} ; \mathbf{r} \mathbf{r}^{\prime}\right) \mathbf{r} \cdot \nabla\left\{\frac{\delta \Delta T_{c}\left[\gamma^{(2)}\right]}{\delta \gamma^{(2)}\left(\mathbf{r}^{\prime} \mathbf{r} ; \mathbf{r}^{\prime} \mathbf{r}\right)}\right\} .
\end{aligned}
$$

In the same way as in the previous paper, ${ }^{8}$ this equation is utilized as a sum rule for $\Delta T_{c}\left[\gamma^{(2)}\right]$. With reference to the approximate form of $T\left[\gamma^{(2)}\right]$ that is proposed by Levy and Ziesche, ${ }^{13}$ we assume the following form for $\Delta T_{c}\left[\gamma^{(2)}\right]$ :

$$
\Delta T_{c}\left[\gamma^{(2)}\right]=\iint \frac{\left.\Delta t_{c}\left(\gamma^{(2)}\right)\right|_{\gamma^{(2)}=\gamma^{(2)}\left(\mathbf{r r}^{\prime} ; \mathbf{r r}^{\prime}\right)}}{\left|\mathbf{r}-\mathbf{r}^{\prime}\right|} \mathrm{d} \mathbf{d} \mathbf{r}^{\prime},
$$

where $\Delta t_{c}\left(\gamma^{(2)}\right)$ is a function of $\gamma^{(2)}$. Substitution of Eq. (A4) into Eq. (A3) leads to

$$
\begin{aligned}
& \iint \frac{1}{\left|\mathbf{r}-\mathbf{r}^{\prime}\right|}\left\{6 \gamma^{(2)} \frac{\partial \Delta t_{c}\left(\gamma^{(2)}\right)}{\partial \gamma^{(2)}}\right. \\
& \left.-7 \gamma^{(2)} \Delta t_{c}\left(\gamma^{(2)}\right)\right\}_{\gamma^{(2)}=\gamma^{(2)}\left(\mathbf{r r}^{\prime} ; \mathbf{r r}^{\prime}\right)} \mathrm{d} \mathbf{r} \mathrm{d} \mathbf{r}^{\prime}=0,
\end{aligned}
$$

where we have neglected the surface integral at infinity. As the necessary condition, we obtain the differential equation with respect to $\Delta t_{c}\left(\gamma^{(2)}\right)$ :

$$
6 \gamma^{(2)} \frac{\partial \Delta t_{c}\left(\gamma^{(2)}\right)}{\partial \gamma^{(2)}}-7 \Delta t_{c}\left(\gamma^{(2)}\right)=0 .
$$

Solving the differential equation [Eq. (A6)], and substituting the solution into Eq. (A4), we arrive at Eq. (13). Note that Eq. (13) just corresponds to the solution that arises if $\Delta t_{c}\left(\gamma^{(2)}\right)$ is restricted to be $\gamma^{(2)}$ raised to a power. ${ }^{13}$

\section{APPENDIX B: PROOF FOR FEATURE (d)}

The total energy functional of the ECS theory is given by ${ }^{8}$

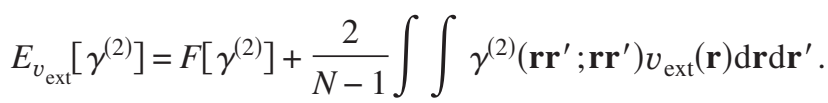

We consider the minimization of $E_{v_{\text {ext }}}\left[\gamma^{(2)}\right]$ within the set of the SSD-representable PDs. For later convenience, this set is denoted as $C^{\prime}$. Using Eqs. (3) and (B1), the minimization is rewritten as

$$
\begin{aligned}
\underset{\gamma^{(2)} \in C^{\prime}}{\operatorname{Min}} E_{v_{\mathrm{ext}}}\left[\gamma^{(2)}\right]= & \operatorname{Min}_{\gamma^{(2)} \in C^{\prime}}\left\{F\left[\gamma^{(2)}\right]\right. \\
& \left.+\frac{2}{N-1} \iint \gamma^{(2)}\left(\mathbf{r r}^{\prime} ; \mathbf{r r}^{\prime}\right) v_{\mathrm{ext}}(\mathbf{r}) \mathrm{d} \mathbf{r} \mathrm{d} \mathbf{r}^{\prime}\right\} \\
= & \underset{\gamma^{(2)} \in C^{\prime}}{\operatorname{Min}}\left\{\operatorname{Min}_{\Psi \rightarrow \gamma^{(2)}}\langle\Psi|H| \Psi\rangle\right\} \\
= & \underset{\Psi \in C_{\Psi}^{\prime}}{\operatorname{Min}}\langle\Psi|H| \Psi\rangle .
\end{aligned}
$$

Here, $C_{\Psi}^{\prime}$ denotes the set of wave functions that yield the PDs belonging to $C^{\prime}$. $C_{\Psi}^{\prime}$, of course, contains not only all SSDs but also other type of wave functions such as $\Phi e^{i f\left(\mathbf{r}_{1}, \mathbf{r}_{2}, \cdots, \mathbf{r}_{N}\right)}$, where $\Phi$ and $f\left(\mathbf{r}_{1}, \mathbf{r}_{2}, \ldots, \mathbf{r}_{N}\right)$ are the SSD and arbitrary function that is real and symmetric, respectively. Taking into account this fact, we have

$$
\underset{\Psi \in C_{\Psi}^{\prime}}{\operatorname{Min}}\langle\Psi|H| \Psi\rangle \leq \underset{\Phi}{\operatorname{Min}}\langle\Phi|H| \Phi\rangle .
$$

The right-hand side of this inequality is just the ground-state energy of the HF equation. Therefore, total energy of the present scheme is lower than that of the HF equation. 
${ }^{1}$ M. Higuchi and K. Higuchi, Phys. Rev. B 69, 035113 (2004).

${ }^{2}$ K. Higuchi and M. Higuchi, Phys. Rev. B 69, 165118 (2004).

${ }^{3}$ K. Higuchi and M. Higuchi, Phys. Rev. B 71, 035116 (2005).

${ }^{4}$ Á. Nagy, S. Liu, and L. Bartolloti, J. Chem. Phys. 122, 134107 (2005).

${ }^{5}$ Á. Nagy, Int. J. Quantum Chem. 106, 1043 (2006).

${ }^{6}$ R. G. Parr and W. Yang, Density-Functional Theory of Atoms and Molecules (Oxford University Press, New York, 1989), Chap. 2.

${ }^{7}$ Many-Electron Densities and Reduced Density Matrices, edited by J. Closlowski (Kluwer, Dordrecht /Plenum, New York, 2000).

${ }^{8}$ M. Higuchi and K. Higuchi, Physica B (Amsterdam) 387, 117 (2007).

${ }^{9}$ P. Ziesche, Phys. Lett. A 195, 213 (1994).

${ }^{10}$ P. Ziesche, Int. J. Quantum Chem. 60, 1361 (1996).

${ }^{11}$ A. Gonis, T. C. Schulthess, J. van Ek, and P. E. A. Turchi, Phys. Rev. Lett. 77, 2981 (1996).

${ }^{12}$ A. Gonis, T. C. Schulthess, P. E. A. Turchi, and J. van Ek, Phys. Rev. B 56, 9335 (1997).

${ }^{13}$ M. Levy and P. Ziesche, J. Chem. Phys. 115, 9110 (2001).

${ }^{14}$ F. Furche, Phys. Rev. A 70, 022514 (2004).

15 Á. Nagy, Phys. Rev. A 66, 022505 (2002).

${ }^{16}$ Á. Nagy and C. Amovilli, J. Chem. Phys. 121, 6640 (2004).

${ }^{17}$ B. Hetényi, L. Brualla, and S. Fantoni, Phys. Rev. Lett. 93, 170202 (2004).

${ }^{18}$ J. K. Percus, J. Chem. Phys. 122, 234103 (2005).

${ }^{19}$ P. W. Ayers, J. Math. Phys. 46, 062107 (2005).

${ }^{20}$ P. W. Ayers and M. Levy, J. Chem. Sci. 117, 507 (2005).

${ }^{21}$ Á. Nagy, Int. J. Quantum Chem. 106, 1043 (2006).

${ }^{22}$ P. W. Ayers, S. Golden, and M. Levy, J. Chem. Phys. 124, 054101 (2006).

${ }^{23}$ M. Higuchi and K. Higuchi, Phys. Rev. A 75, 042510 (2007).

${ }^{24}$ B. Hetényi and A. W. Hauser, Phys. Rev. B 77, 155110 (2008).

${ }^{25}$ P. Hohenberg and W. Kohn, Phys. Rev. 136, B864 (1964).

${ }^{26}$ W. Kohn and L. J. Sham, Phys. Rev. 140, A1133 (1965).

${ }^{27}$ A. Szabo and N. Ostlund, Modern Quantum Chemistry, 1st ed. (Dover, New York, 1996).

${ }^{28}$ Springer Handbook of Atomic, Molecular, and Optical Physic, edited by G. W. F. Drake (Springer, New York, 2006).

${ }^{29}$ A. J. Coleman, Rev. Mod. Phys. 35, 668 (1963).

${ }^{30}$ A. J. Coleman, in The Force Concept in Chemistry, edited by B. M. Deb (Van Nostrand Reinhold, New York, 1981), p. 418.
${ }^{31}$ A. J. Coleman and V. I. Yukalov, Reduced Density Matrices: Coulson's Challenge (Springer-Verlag, Berlin, 2000).

${ }^{32}$ The Fundamentals of Electron Density, Density Matrix and Density Functional Theory in Atoms, Molecules and the Solid States, edited by N. I. Gidopoulos and S. Wilson (Kluwer, New York, 2003).

${ }^{33}$ E. R. Davidson, Chem. Phys. Lett. 246, 209 (1995).

${ }^{34}$ S. Kh. Samvelyan, Int. J. Quantum Chem. 65, 127 (1997).

${ }^{35}$ M.-E. Pistol, Chem. Phys. Lett. 400, 548 (2004).

${ }^{36}$ M.-E. Pistol, Chem. Phys. Lett. 417, 521 (2006).

${ }^{37}$ M.-E. Pistol, Chem. Phys. Lett. 422, 363 (2006).

${ }^{38}$ M.-E. Pistol, Chem. Phys. Lett. 431, 216 (2006).

${ }^{39}$ P. W. Ayers and M. Levy, Chem. Phys. Lett. 415, 211 (2005).

${ }^{40}$ P. W. Ayers and E. R. Davidson, Int. J. Quantum Chem. 106, 1487 (2006).

${ }^{41}$ P. W. Ayers, Phys. Rev. A 74, 042502 (2006).

${ }^{42}$ P. W. Ayers and S. Liu, Phys. Rev. A 75, 022514 (2007).

${ }^{43}$ P. W. Ayers and E. R. Davidson, Adv. Chem. Phys. 134, 443 (2007).

${ }^{44}$ M.-E. Pistol, Chem. Phys. Lett. 449, 208 (2007).

${ }^{45}$ P. Gori-Giorgi and A. Savin, Philos. Mag. 86, 2643 (2006).

${ }^{46}$ The HF kinetic energy means the kinetic energy calculated within the HF approximation.

${ }^{47}$ J. E. Harriman, Phys. Rev. A 24, 680 (1981).

${ }^{48}$ S. E. Koonin, Computational Physics (Addison-Wesley, Reading, MA, 1986).

${ }^{49}$ It is noted that if the present scheme is applied to the open-shell atoms, we further have to consider the effect of the nonspherical distribution of electrons, which is never negligible. (For example, see Refs. 50-54.)

${ }^{50}$ J. F. Janak and A. R. Williams, Phys. Rev. B 23, 6301 (1981).

${ }^{51}$ F. W. Kutzler and G. S. Painter, Phys. Rev. Lett. 59, 1285 (1987).

${ }^{52}$ M. Miyasita, K. Higuchi, A. Narita, and M. Higuchi, Mater. Trans. 49, 1893 (2008).

${ }^{53}$ A. D. Becke, J. Chem. Phys. 117, 6935 (2002).

${ }^{54}$ K. Higuchi and M. Higuchi, Phys. Rev. B 74, 195122 (2006).

${ }^{55}$ A. V. Bunge and R. O. Esquivel, Phys. Rev. A 34, 853 (1986).

${ }^{56}$ E. Clementi and C. Roetti, At. Data Nucl. Data Tables 14, 177 (1974).

${ }^{57}$ A. Veillard and E. Clementi, J. Chem. Phys. 49, 2415 (1968). 